\title{
Evaluating the Combined Impact of Node Architecture and Cloud Workload Characteristics on Network Traffic and Performance/Cost
}

\author{
Diman Zad Tootaghaj ${ }^{1}$, Farshid Farhat ${ }^{1}$, Mohammad Arjomand ${ }^{1}$, Paolo Faraboschi ${ }^{2}$ \\ Mahmut Taylan Kandemir ${ }^{1}$, Anand Sivasubramaniam ${ }^{1}$, Chita R. Das ${ }^{1}$ \\ ${ }^{1}$ The Pennsylvania State University, ${ }^{2}$ Hewlett-Packard Labs \\ $\{d x z 149$, fuf111, arjomand, kandemir, das, anand\}@cse.psu.edu, \{paolo.faraboschi\}@hp.com
}

\begin{abstract}
The combined impact of node architecture and workload characteristics on off-chip network traffic with performance/cost analysis has not been investigated before in the context of emerging cloud applications. Motivated by this observation, this paper performs a thorough characterization of twelve cloud workloads using a full-system datacenter simulation infrastructure. We first study the inherent network characteristics of emerging cloud applications including message inter-arrival times, packet sizes, inter-node communication overhead, selfsimilarity, and traffic volume. Then, we study the effect of hardware architectural metrics on network traffic. Our experimental analysis reveals that (1) the message arrival times and packet-size distributions exhibit variances across different cloud applications; (2) the inter-arrival times imply a large amount of self-similarity as the number of nodes increase; (3) the node architecture can play a significant role in shaping the overall network traffic; and finally, (4) the applications we study can be broadly divided into those which perform better in a scale-out or scale-up configuration at node level and into two categories, namely, those that have long-duration, low-burst flows and those that have short-duration, high-burst flows. Using the results of (3) and (4), the paper discusses the performance/cost trade-offs for scale-out and scale-up approaches and proposes an analytical model that can be used to predict the communication and computation demand for different configurations. It is shown that the difference between two different node architecture's performance per dollar cost (under same number of cores system wide) can be as high as $\mathbf{1 5 4}$ percent which disclose the need for accurate characterization of cloud applications before wasting the precious cloud resources by allocating wrong architecture. The results of this study can be used for system modeling, capacity planning and managing heterogeneous resources for large-scale system designs.
\end{abstract}

Keywords-Workload characteristics; Performance/Cost analysis

\section{INTRODUCTION}

Cloud applications, ranging from interactive query-based jobs to high performance computing applications are driving the development of current datacenters that are composed of tens of thousands of nodes to handle extremely large amounts of data processing and operation execution. Efficiently executing these applications requires having sufficient computational, storage, and network bandwidth resources. For example, many Map-Reduce jobs, have bursty network communications during the map and data shuffling phases [1, 2]. Similarly, many cloud applications and high-performance computing (HPC) applications need high communication bandwidth [3].

While every other aspect of datacenter performance have been improved drastically, network bandwidth and latency has been a source of performance degradation in cloud computing for years [3, 4]. High network latency abandons any hope for getting performance benefit on large number of machines and makes the scale-out approach [5, 6] challenging for networkintensive applications. On the other hand, the ever-growing data sizes in Big Data era, that cannot be processed in a single server or reside in memory makes distributed processing unavoidable. Therefore, given a workload and a set of computational and storage resources, an in-depth study of network traffic demand, can be the key to reducing costs and boosting performance. Analyzing the combined impact of hardware architecture and workload can help cloud providers in designing performance/cost efficient datacenters. There exists prior works $[7,8,9]$ that studied workload characteristics and network traffic in datacenters. High-performance computing (HPC) nodes produce higher traffic bursts compared to lowperformance computing nodes and on the other hand can handle part of traffic within cores using inter-core interconnection network. In addition to node architecture, the other factor that shapes message traffic is the inherent temporal characteristics of the workload. Most of the previous studies on network topology exploration employ either synthetic traffic or non-real workloads [10, 11, 12]. However, the hardware architectures employed by datacenters and workloads are changing rapidly; as a result, the traffic patterns and application characteristics taken from prior studies may not be applicable to current systems. To the best of our knowledge, none of these studies investigated the impact of node architecture and real workload characteristics on inter-node network traffic.

In addition, conventional trend in academia and industry suggests using a cluster of commodity servers, which is called scale-out for cloud computing [5]. Appuswamy et al. [13] suggest adding more resources to a single server, which is called scale-up approach. In this paper, we discuss performance/cost trade-offs for these controversial approaches for different cloud applications. We consider different types of cloud workloads ranging from those that represent MapReduce and client-server paradigms in CloudSuite [14, 15, 16] to memory-intensive large-scale scientific computing benchmarks in Mantevo [17], and network-intensive parallel graph algorithms in Graphlab [18, 19].

The main goal of this paper is to study the combined impact of node architecture (the number of cores, intra-node network, cache/memory hierarchy) and cloud workload characteristics on inter-node network traffic as well as performance/cost analysis. Using a set of twelve cloud workloads with different characteristics, the specific research questions that we strive to answer can be summarized as follows:

- What is the message inter-arrival times' distribution and 
packet size distribution of different cloud workloads to regenerate the same traffic pattern?

- What factors affect the burstiness of network traffic in the cloud? How much role does the node architecture play in order to design better network buffer managements?

- What is the ratio of communication time over computation time for cloud workloads and how does it scale with respect to the volume of data to provision network capacity for each cloud workload?

- How does the bandwidth requirements of different cloud benchmarks change with respect to the basic node parameters such as the number of cores and cache/memory capacity? This observation helps a designer to provide enough network resources when configuring resources to the current datacenter architecture.

- How much is the performance/cost of different workloads for scale-out or scale-up approaches? which helps us to find the right way to expand the datacenter for the running workloads.

- What is the impact of node architecture on network traffic and performance/cost? Which of scale-out or scale-up approaches is better for each studied cloud workloads?

We carried out a series of experimental study using a fullsystem simulation that gives us exploring the effect of hardware parameters on the system performance. It also helps to isolate traffic pattern of different applications [20]. We used a set of modern workloads in cloud study including Cloudsuite [14, 15, 16], Mantevo [17] and Graphlab [18, 19] benchmark suites. We also generalized experimental study for largesized datacenters to ease capacity planning and performance optimization. We can derive the following results from our experimental study:

- The studied workloads can be categorized into three different groups: memory-intensive applications, network bandwidthintensive applications, and network latency-sensitive applications. Applications belonging to different groups require different design optimization considerations.

- Running network bandwidth-intensive or latency-sensitive applications on a scale-out hardware architecture does not give us much performance/cost benefit compared to the scaleup approach. On the other side, memory-intensive applications scale well and the scale-out approach gives better performance/cost.

- For most of the studied cloud benchmarks, the packet interarrival times follow lognormal or extreme value distribution, which is a long-tailed distribution and causes self-similarity in network traffic. Self-similar traffic increases queue length in the network and this degrades the performance of the system. There exist prior works on how to perform buffer management in the presence of self-similarity in network traffic [21]. Self-similar traffic is quantified by a Hurst parameter. Larger Hurst parameter represents higher selfsimilarity for a flow and more possibility to congest the network. Consequently, if we knew the self-similarity quantity of each application in advance, it would be possible to employ better buffer management and achieve better performance. It was observed that increasing the dataset size and the number of nodes increase the hurst parameter for self-similarity.

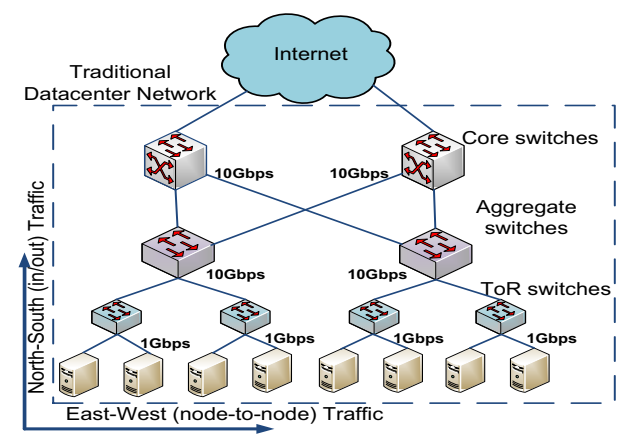

Fig. 1: A three-layer datacenter architecture.

- We used the parameters of AMD Opteron server processors $[22,23]$ and observed that the node architecture (e.g., number of cores, cache/memory capacity) plays an important role on bandwidth; for instance, the effect of node configuration (under the same number of cores system wide) can be as much as $40.7 \%$ on execution time and $154 \%$ on performance per dollar; and the effect of network configurations on execution time can be as much as $74 \%$.

The rest of this paper is structured as follows. Section II discusses the motivation and background behind this work. Section III explains experimental setup. Section IV studies network characteristics of studied workloads. Section V describes the evaluation results. Section VI summarizes the potential implications of our major findings. Section VII studies related work and finally Section VIII concludes the paper.

\section{MOTIVATION AND BACKGROUND}

\section{A. Motivation}

With the rapid increase in consumer demands and increasing complexity of the cloud applications, datacenter designers are steadily revisiting design of nodes and network architectures in datacenters. As both applications and node architectures are rapidly changing, analyzing the impact of both on network traffic is becoming increasingly important. Large cloud computing vendors such as Facebook, Amazon, and Google rely heavily on traditional network structures, which consist of two or three levels of switch hierarchy shown in Figure $1[7,11]$.

As technology improves, more and more cores are being embedded into a single node and the communication bandwidth requirement of datacenters keeps increasing. As pointed out by prior studies $[3,4,10]$, one of the main limitations of today's large-scale computing is the network communication latency and bandwidth. There have been several prior studies attempted to find better network topologies and routing algorithms in datacenters $[10,11]$. However, to our knowledge, there is no prior work that characterizes the combined impact of machine architecture/parameters and cloud application characteristics on the network traffic, performance/cost of scalability. Such a study could help us identify the application's specific bottlenecks and ultimately reach cost-efficient datacenter designs for different characteristics of cloud workloads. In addition, accurate workload characterization is important for system modeling and capacity planning. Specifically, the capacity planning for large-scale system design needs more accurate modeling and understanding of workload requirement and behavior. Operating and running large computation in large 
scale is expensive and large cloud vendors have to know how to expand node architecture and network architecture resources to satisfy the ever-increasing job workload demands. Recent trend in academia and industry is to use large number of commodity servers for cloud computing [5, 6]. However, based on network communication pattern, different workloads have completely different performance metrics for scale-up and scale-out approach. Therefore, in this paper, we conduct a simulation-based study, using a full-system simulator, that simplifies design space exploration, to perform such evaluations in smaller scale to find out the network traffic pattern of these applications in small-scaled systems and generalize it to large-scale ones. To this end, we derive an analytical model and use non-linear regression analysis to find the accuracy of the predicted model.

\section{B. Background}

Traditional datacenter architectures consist of two or three layers structured as a tree, where higher layer switches have high capacity with more ports but more expensive. In order to reduce the cost, most datacenter network designers use oversubscription factor of 2.5:1 to 8:1 [10]. An over-subscription of $n: 1$ means that in worst case traffic patterns, only $1 / n$ of the total communication bandwidth is available to different nodes in that traffic pattern. The concurrent flows for each cloud application plays an important role on how much we can over-subscribe a network architecture. For example, if an application has all-to-all communication pattern, oversubscribing the network would degrade the performance since the network would be the bottleneck resource. Concurrent flow analysis can guide system administrative on choosing the appropriate factor for over-subscription. Figure 1 shows an example of a three-layer datacenter network architecture, where the servers at each rack are connected to the Top of Rack (ToR) switches with 1 Gbps Ethernet links. ToR switches are connected to the aggregate switches using 10 Gbps links and finally aggregate switches are connected to the core switches using a fat-tree topology. It is important to provision enough bandwidth for each link in the hierarchy when we scale-up or scale-out the servers.

Network traffic can be distinguished as either node-to-node (East-West) communication or in-out traffic (North-South). North-South traffic patterns happen in client-server applications, where the clients from the internet side (Figure 1) send queries to the servers and get responses. The communication pattern in North-South traffic patterns is mainly between client and servers and the servers do not have communication among themselves. While, in East-West traffic patterns, the communication is mainly among servers. The client-server and interactive query-base applications in Cloudsuite have NorthSouth traffic pattern and the servers never communicate with each other. However, most scientific benchmarks like graph algorithms from Graphlab and Mantevo benchmarks in our experiments have East-West traffic behavior.

Provisioning for East-West traffic is more difficult than North-South traffic, because North-South traffic challenges can be solved by means of load balancing and replication techniques. However, it has been extensively discussed that East-West network bandwidth and latency is the main source of performance degradation in large-scale computing [3]. Therefore, we focus on East-West traffic patterns as dominant behavior in datacenters.

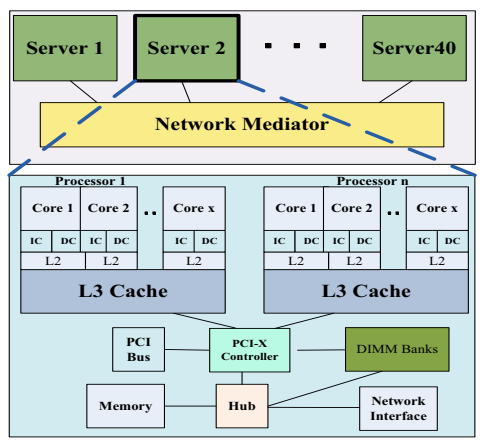

Fig. 2: High-level view of the simulated architecture.

TABLE I: Node parameters and their default values.

\begin{tabular}{ll}
\hline \multicolumn{2}{c}{ Processor parameters and their default values } \\
\hline Clock frequency & $2400 \mathrm{MHz}$ \\
Main Memory latency & 150 cycles \\
L1 caches & Split I and D, 64kB private, 2-way, 64B, LRU, \\
& write-through, 2-cycle hit \\
L1 TLB & Split I and D, 4kB private, 32 entries, 2-cycle \\
& latency \\
L2 cache & Unified, 1MB private, inclusive, 4-way, 64B, LRU, \\
& write-back, 10-cycle hit \\
L3 cache & 4MB shared, NUCA, inclusive, 16-way, 64B, LRU, \\
& write-back, 4-cycle tag, 10-cycle data hit, 40-cycle \\
& CPU to L2 \\
\hline
\end{tabular}

\section{EXPERIMENTAL SETUP}

In this section, we describe the simulation setup and different node and network architectures that we tested. To capture fine-grained network traffic pattern (in the granularity of micro second) for any specific workload, we employed COTSon fullsystem simulator [24], that is based on AMD Simnow [25]. Simnow simulates an entire server machine including cores, memory, I/O and network interfaces. To simulate multiple nodes, COTSon provides networking for Simnow and uses a parallel discrete-event model, allowing the simulation of multi-core clusters. The simulator uses a mediator to provide a network interface between nodes (or servers) [26], and it uses Ubuntu 64-bit x-86 3.5.0-23-generic Linux as the operating system.

\section{A. Simulated Configurations}

1) Node Architectures: We used the parameters of an AMD Opteron server processor as a baseline in our simulations [22]. Table I gives the main architectural parameters of the baseline system configuration. Each core has its own private instruction and data L1 and L2 caches and an L3 cache is shared among all the cores in the processor. The memory, L3 cache, network interface and DIMM banks are connected through a hub device. Figure 2 shows the target architecture simulated in our experiments. Table II shows the different node architectures varied in this work. We changed the number of servers from 1 to 40 (scale-out degree) and the number of cores per node from 1 to 6 (scale-up degree).

2) Network Architectures: We used three different network architectures with different bandwidth, latency and congestion parameter, as shown in Table III. We used the standard ToR switches that contain 48 GigE ports. This table specifies each network configuration in terms of maximum available bandwidth of links (in Gbps), minimum latency of the switches (in $\mu s$ ) and a congestion factor (cfactor) that shows the queuing latency of the switches. 
TABLE IV: Evaluated workloads.

\begin{tabular}{ll}
\hline Application & Description \\
\hline pagerank (pgrnk) & An algorithm used by Google that computes the pagerank of each vertex in the graph. \\
format convert (fcvt) & Converts a graph from a specific format, for example snap, tsv, adj or binsv4 to another format. \\
undirected triangle count (udtc) & Counts the total number of triangles in a graph using the algorithm in [28]. \\
directed triangle count (dtc) & Counts the total number of directed triangles in the graph. \\
kcore decomposition (kcore) & $\begin{array}{l}\text { Iteratively computes sub graphs of k cores in a given graph. A sub graph is called k-core if and only if all vertices in the sub } \\
\text { graph is at least of degree k. }\end{array}$ \\
connected components (cc) & Computes all connected components and the number of vertices in that component for a given graph. \\
approximate diameter (apxr) & Computes the approximate diameter of a given graph using the proposed algorithm in [29]. \\
$\begin{array}{l}\text { Data Serving (dsrv) } \\
\text { memcached (mem) }\end{array}$ & $\begin{array}{l}\text { A benchmark from Cloudsuite [16] for data store systems that is used for large-scale web applications. } \\
\text { A client-server architecture for distributed memory caching. The servers keep key-value stores and clients send query for these }\end{array}$ \\
key- & A part of Mantevo benchmarks and is an mpi implementation of molecular dynamics algorithms which is used in material science. \\
MiniFE & An approximation to an unstructured implicit finite element code. The application was configured with MPI support. \\
HPCCG & Implements a Conjugate Gradient solver, where the coefficient matrix is stored in a sparse matrix format. \\
\hline
\end{tabular}

TABLE II: Different node architectures considered in this work.

\begin{tabular}{|c|l|l|l|}
\hline Architecture & L3 size/latency & Memory Size & cores \\
\hline Default & $4 \mathrm{MB} / 40$ cycles & $512 \mathrm{M}$ & 1 \\
\hline Double Cache & $8 \mathrm{MB} / 60$ cycles & $512 \mathrm{M}$ & 1 \\
\hline Double memory & $4 \mathrm{MB} / 40$ cycles & $2048 \mathrm{M}$ & 1 \\
\hline Dual-core & $8 \mathrm{MB} / 60$ cycles & $1024 \mathrm{M}$ & 2 \\
\hline 4-core & $16 \mathrm{MB} / 80$ cycles & $2024 \mathrm{M}$ & 4 \\
\hline 6-core & $24 \mathrm{MB} / 100$ cycles & $6144 \mathrm{M}$ & 6 \\
\hline
\end{tabular}

TABLE III: Different network architectures.

\begin{tabular}{|c|l|l|l|}
\hline Network Parameter & Network 1 & Network 2 & Network 3 \\
\hline Ports & $48^{*} 1 \mathrm{GbE}$ & $48^{*} 1 \mathrm{GbE}$ & $48^{*} 1 / 10 \mathrm{GbE}$ \\
\hline Maximum Bandwidth & $1 \mathrm{Gbps}$ & $1 \mathrm{Gbps}$ & $100 \mathrm{Mbps}$ \\
\hline Latency & $4 \mu \mathrm{s}$ & $40 \mu \mathrm{s}$ & $4 \mu \mathrm{s}$ \\
\hline cfactor & 2 & 10 & 10 \\
\hline
\end{tabular}

\section{B. Evaluated Workloads}

We used a diverse set of cloud applications ranging from interactive query-based to high-performance scientific workloads. The workload set include seven programs from Graphlab benchmark suite [18], two programs from Cloudsuite benchmarks [14], and three programs from Mantevo benchmarks [17]. The twelve benchmark programs used in this work and short descriptions can be summarized in Table IV ${ }^{1}$. The graph analytic toolkit from Graphlab has different applications that analyze a graph structure. As input for these applications, we used Stanford large network dataset collections [27] which include different graphs from social networks and communication networks. We also used two benchmarks from Cloudsuite, that are based on online applications. The Graphlab uses Open MPI and Hadoop implementation of Map-Reduce to perform graph computations on large graphs. The Mantevo benchmarks are open-source mini-applications to analyze, predict and improve HPC systems.

\section{Evaluation Methodology}

To quantify the impact of node and network architectures on the network traffic pattern and overall workload performance, we evaluate five different scenarios:

1) Changing the number of cores per socket, and increasing the total cache/memory capacity, we evaluate the effect of scale-up on network communication as well as performance/cost trade-offs.

\footnotetext{
${ }^{1}$ The names in parentheses are abbreviations used in this paper
}

2) Changing the capacity of LLC per node, we determine the memory-intensity of the applications.

3) Changing the total number of nodes in the system, we evaluate the network traffic pattern as well as the traffic self similarity metrics.

4) Changing the network bandwidth and latency, we determine the sensitivity of the applications to the network configurations.

5) We compare the scale-out of 40 single-core machines with the scale-up of 12 quad-core machines for different applications.

\section{Metrics}

The metrics used to evaluate the impact of node configuration and workload characteristics on network traffic are:

- Communication over computation overhead gives the overheads incurred due to parallel execution. This metric can be used to evaluate the communication overhead experienced when running a given benchmark in parallel.

- Packet inter-arrival times and packet size distribution These metrics, capture the burstiness of the incoming traffic and if known by a designer, he/she can regenerate the traffic pattern for synthetic traffic generation. Furthermore, knowing these metrics in advance help us to provision network buffers and have better bandwidth allocation for different applications.

- Self similarity indicates the burstiness of the network traffic for different benchmarks.

- Bandwidth requirement gives the amount of bandwidth needed between different nodes for a given dataset and hardware architecture.

- Concurrent flows shows the maximum number of concurrent flows in an application/workload during execution. This metric can be used to predict the required bisection bandwidth of each application to choose appropriate oversubscription factor. For example, if an application has an all-to-all traffic pattern (highest number of the concurrent flows), over-subscribing the network degrades the overall system performance.

- Performance per dollar cost indicates how much performance increase we will get for each dollar we spend on system configuration. 
TABLE V: Hurst parameter estimation for different number of nodes.

\begin{tabular}{|c|c|c|c|c|c|c|}
\hline 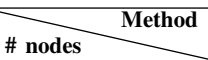 & aggvar & boxper & diffvar & peng & per & $\mathbf{R} / \mathbf{S}$ \\
\hline 5 & 0.478 & 0.454 & 0.253 & 0.448 & 0.430 & 0.571 \\
\hline 10 & 0.484 & 0.461 & 0.388 & 0.485 & 0.441 & 0.577 \\
\hline 15 & 0.541 & 0.472 & 0.453 & 0.580 & 0.453 & 0.601 \\
\hline 20 & 0.584 & 0.480 & 0.666 & 0.597 & 0.462 & 0.653 \\
\hline 40 & 0.634 & 0.520 & 0.712 & 0.621 & 0.482 & 0.664 \\
\hline
\end{tabular}

\section{Network Characteristics' of Applications}

\section{A. Inter-arrival Times and Self Similarity}

Most prior works on datacenter networks use synthetic traffic with exponential distribution to tune network and/or application parameters. However, in our experiments the interarrival time of our cloud applications follow a long-tailed distribution [30], that can result in performance degradation, unless we provision enough buffers in the network.

We found that, for most of the evaluated graph analytic benchmarks, the generalized extreme value distribution ${ }^{2}$ gives the best fit for packet inter-arrival times' distribution. We further observed that, the lognormal distribution, which has two degrees of freedom, seems to be a good fit for Graphlab benchmarks. Table VI lists the parameters for each distribution using the maximum likelihood estimation and the normalized Euclidean distance (error) we obtain for each distribution.

Similar to our finding, prior studies [31, 32] have shown that the traffic pattern of Ethernet follows a self-similar distribution which degrades network performance. Self-similar processes can be described using long-tailed distributions like Pareto, lognormal, and Weibull distribution. Self-similarity in networks is not a pleasant phenomenon, since network performance can degrade with increasing self-similarity due to, primarily, the queue length increase. The effect of self similarity on network performance is studied by Park et al. [33]. Taqqu et al. [30] proposed several strategies to estimate the self similarity parameter. We follow [30] and evaluate Hurst parameter as an estimation of self similarity. Table $\mathrm{V}$ gives the result of this estimation using the different strategies discussed in [30]. It is observed that increasing the number of nodes increases the self-similarities. Consequently, network designer need to provision more buffers when running the workloads on large number of machines. Those flows, which have higher Hurst parameter, fill up the networks buffers and non-self similar traffics would be discarded. By knowing the Hurst parameter for different flows, a designer can estimate queue length for network buffers and potentially design better buffer management system.

\section{B. Packet Size Distribution}

In addition to the message inter-arrival times, packet size distribution has to be known for regeneration of the same traffic pattern. For instance, one needs to consider a higher network bandwidth and more buffers in networks with larger packet sizes. Here, we report the analysis results of packet size distribution of our benchmarks. The simulation results, in Figure 3 show that packet size distribution for the benchmarks

\footnotetext{
${ }^{2}$ Generalized extreme value distribution has three degrees of freedom and can result a better fit with less error
}

which use Map-Reduce framework follow a bimodal distribution with two peaks in 66 bytes and 1514 bytes, that is the maximum Ethernet packet size. Pagerank, approximate diameter, connected component, format convert, directed triangle count and undirected triangle count are the applications, which have larger packet sizes with a bimodal distribution. However, for web applications, packet sizes have a multi-modal distribution with smaller packets. Memcached, data serving and kcore are three applications that have a multi-modal packet size distribution with fewer packet sizes. Knowing the packet size distribution of these workloads helps us to perform better traffic and quality of service (QoS) management in the network only by extracting the packet size in the header. Smaller packets can get higher priority than larger packets. Also the high frequency of large packets (1514 bytes) shows the room for improving the packet control protocol in current datacenter networks to allow transmitting higher sizes of packets.

\section{Concurrent Flow Analysis}

Different applications generate different ranges of long-term or short-term traffic flows. Datacenter networks should be able to tolerate large traffic bursts, provide low latency for short flows and high utilization for long flows. In addition, the number of concurrent flows in an application gives us a metric to determine how much the bisection bandwidth is being utilized in the network. Computing the number of concurrent flows for an application helps us to have a better understanding of the bisection bandwidth utilization of that application. This helps an administrator to determine how to over-subscribe the network for cost reduction. To compute concurrent flows of each application, we use the steps given in Algorithm 1. Using the time and size of packets exchanging between two nodes, Algorithm 1 finds all the longest contiguous intervals that these two nodes send receive. The threshold of having a connection is the mean rate of all communications, i.e., if packet size per its inter-arrival time is higher than the mean (threshold), that interval is considered as a flow between these two nodes.

Table VII shows the comparison of concurrent flows from the master node to all slave nodes for each application running on a 10 nodes. We observed that, kcore, Data serving, and directed triangle count have the most concurrent flows. Therefore, running these applications on the same rack can be expected to reduce congestion in the higher layers of the datacenter hierarchy. In addition, we compute average flow duration of different benchmarks, some benchmarks, like kcore, data serving, and memcached, have long-term flow duration which makes them suitable fit for hybrid architecture (circuit and packet switching) networks where long-term duration flows can go through the circuit switching network. Other benchmarks, with short-term flows, need to go through the packet switching network part of the hybrid network.

\section{Evaluation}

\section{A. Communication over Computation Overhead}

We start by studying the communication overhead with different number of nodes. We change the number of nodes from 1 to 40 and measure the total execution time it takes for the server nodes to perform pagerank graph computation. We chose pagerank benchmark as a representative of graph applications, because it has more inter-node communication overhead. We recall that that the total execution time taken 


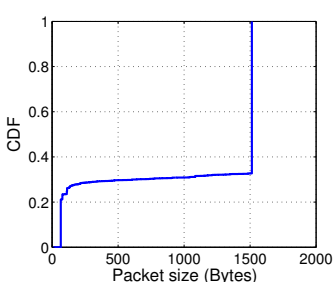

(a) pgrnk.

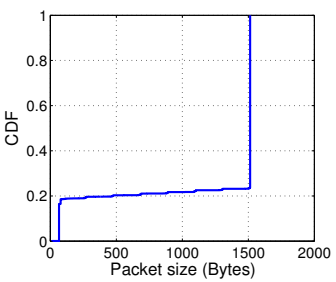

(e) apxr, cc, fcvt, dtc, udtc.

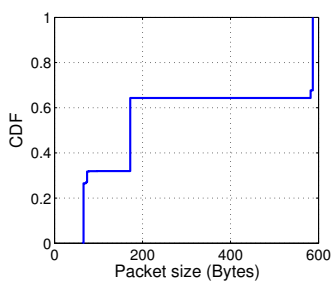

(b) mem.

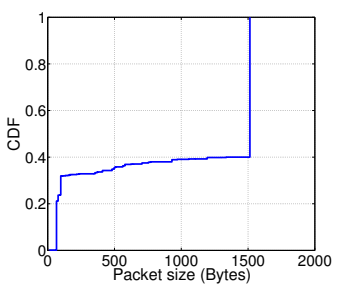

(f) MiniFE.

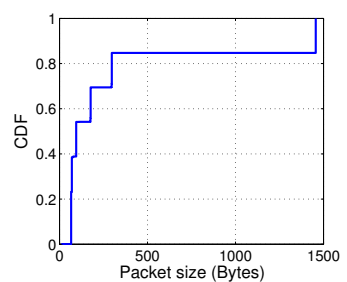

(c) dsrv.

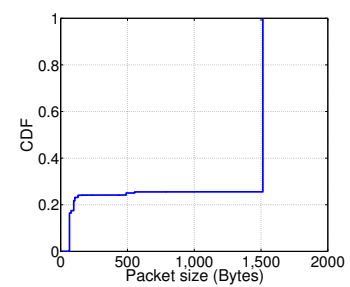

(g) HPCCG.

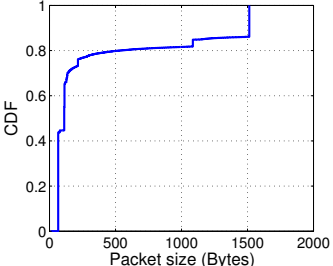

(d) kcore.

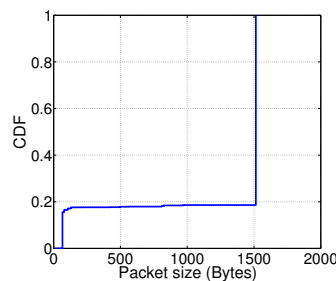

(h) CoMD

Fig. 3: CDF of packet sizes in the graph analytic and web benchmarks.

TABLE VI: Error and best fitting parameters of different distributions for packet inter-arrival times in different benchmarks.

\begin{tabular}{|c|c|c|c|c|c|}
\hline $\begin{array}{ll}\text { Application } & \text { Distribution }\end{array}$ & exponential & gamma & gev & weibull & lognormal \\
\hline Pagerank & $\begin{array}{l}\text { Error: } 0.030 \\
\text { Mean: } 0.6711\end{array}$ & $\begin{array}{c}\text { Error: } 0.0058 \\
\text { Shape: } 0.1516 \\
\text { Scale: } 0.435\end{array}$ & $\begin{array}{c}\text { Error: } 9.5 \mathrm{e}-4 \\
\text { Shape: } 1.85, \text { Scale: } 2.76 \mathrm{e}-5 \\
\text { Location: } 1.47 \mathrm{e}-5\end{array}$ & $\begin{array}{c}\text { Error: } 7.1 \mathrm{e}-4 \\
\text { Scale: } 1.51 \mathrm{e}-4 \\
\text { Shape: } 0.34\end{array}$ & $\begin{array}{l}\text { Error: } 5.7 \mathrm{e}-4 \\
\text { Mean: } \mathbf{- 1 0 . 0 8 2 8} \\
\text { SD: } \mathbf{2 . 3 9}\end{array}$ \\
\hline connected components & $\begin{array}{l}\text { Error: } 0.0386 \\
\text { Mean: } 0.0086\end{array}$ & $\begin{array}{l}\text { Error: } 0.0063 \\
\text { Shape: } 0.138 \\
\text { Scale: } 0.062\end{array}$ & $\begin{array}{c}\text { Error: } 5.4 \mathrm{e}-4 \\
\text { Shape: } 1.397, \text { Scale: } 1.93 \mathrm{e}-5 \\
\text { Location: } 1.30 \mathrm{e}-5\end{array}$ & $\begin{array}{l}\text { Error: } 8.6 \mathrm{e}-4 \\
\text { Scale: } 1.05 \mathrm{e}-4 \\
\text { Shape: } 0.32\end{array}$ & $\begin{array}{l}\text { Error: } 6.1 \mathrm{e}-4 \\
\text { Mean: }-10.4062 \\
\text { SD: } 2.20\end{array}$ \\
\hline data serving & $\begin{array}{l}\text { Error: } 9.3404 \\
\text { Mean: } 1.91\end{array}$ & $\begin{array}{l}\text { Error: } 0.0308 \\
\text { Shape: } 0.158 \\
\text { Scale: } 12.07\end{array}$ & $\begin{array}{c}\text { Error: } 0.1240 \\
\text { Shape: } 4.43, \text { Scale: } 0.459 \\
\text { Location: } 0.1035\end{array}$ & $\begin{array}{l}\text { Error: } 0.0286 \\
\text { Scale: } 0.347 \\
\text { Shape: } 0.213\end{array}$ & $\begin{array}{l}\text { Error: } 0.0141 \\
\text { Mean: } \mathbf{- 4 . 1 7 8} \\
\text { SD: } \mathbf{8 . 4 0}\end{array}$ \\
\hline kcore & $\begin{array}{l}\text { Error: } 0.0079 \\
\text { Mean: } 0.0028\end{array}$ & $\begin{array}{l}\text { Error: } 0.0045 \\
\text { Shape: } 0.397 \\
\text { Scale: } 0.007\end{array}$ & $\begin{array}{c}\text { Error: } 5.7 \mathrm{e}-4 \\
\text { Shape: } 0.805 \text {, Scale: } 0.00047 \\
\text { Location: } 0.00039\end{array}$ & $\begin{array}{c}\text { Error: } 0.0019 \\
\text { Scale: } 0.0012 \\
\text { Shape: } 0.59\end{array}$ & $\begin{array}{l}\text { Error: } 0.0013 \\
\text { Mean: }-7.54 \\
\text { SD: } 1.69\end{array}$ \\
\hline memcached & $\begin{array}{l}\text { Error: } 9.9728 \\
\text { Mean: } 2.037\end{array}$ & $\begin{array}{l}\text { Error: } 0.0214 \\
\text { Shape: } 0.107 \\
\text { Scale: } 19.08\end{array}$ & $\begin{array}{c}\text { Error: 0.0096 } \\
\text { Shape: } 7.76, \text { Scale: } 9.27 \mathrm{e}-5 \\
\text { Location: } 1.29 \mathrm{e}-5\end{array}$ & $\begin{array}{l}\text { Error: } 0.0156 \\
\text { Scale: } 0.0303 \\
\text { Shape: } 0.163\end{array}$ & $\begin{array}{l}\text { Error: } 0.0125 \\
\text { Mean: }-6.834 \\
\text { SD: } 7.94\end{array}$ \\
\hline directed triangle count & $\begin{array}{l}\text { Error: } 0.0282 \\
\text { Mean: } 0.0063\end{array}$ & $\begin{array}{l}\text { Error: } 0.0059 \\
\text { Shape: } 0.1386 \\
\text { Scale: } 0.0456\end{array}$ & $\begin{array}{l}\text { Error: } 4.9 \mathrm{e}-4 \\
\text { Shape: } 1.12 \text {, Scale: } 1.50 \mathrm{e}-5 \\
\text { Location: } 1.18 \mathrm{e}-5\end{array}$ & $\begin{array}{l}\text { Error: } 8 \mathrm{e}-4 \\
\text { Scale: } 7.117 \mathrm{e}-5 \\
\text { Shape: } 0.34\end{array}$ & $\begin{array}{l}\text { Error: } 5.5 \mathrm{e}-4 \\
\text { Mean: } \mathbf{- 1 0 . 6 7 2} \\
\text { SD: } 1.94\end{array}$ \\
\hline undirected triangle count & $\begin{array}{l}\text { Error: } 0.0345 \\
\text { Mean: } 0.0077\end{array}$ & $\begin{array}{l}\text { Error: } 0.0062 \\
\text { Shape: } 0.138 \\
\text { Scale: } 0.055\end{array}$ & $\begin{array}{c}\text { Error: 6.9e-4 } \\
\text { Shape: 1.101, Scale: } 1.62 \mathrm{e}-5 \\
\text { Location: } 1.37 \mathrm{e}-5\end{array}$ & $\begin{array}{l}\text { Error: } 8.7 \mathrm{e}-4 \\
\text { Scale: } 8.76 \mathrm{e}-5 \\
\text { Shape: } 0.34\end{array}$ & $\begin{array}{l}\text { Error: 7.1e-4 } \\
\text { Mean: } \mathbf{- 1 0 . 4 9} \\
\text { SD: } 1.97\end{array}$ \\
\hline approximate diameter & $\begin{array}{l}\text { Error: } 0.0661 \\
\text { Mean: } 0.014\end{array}$ & $\begin{array}{l}\text { Error: } 0.0072 \\
\text { Shape: } 0.127 \\
\text { Scale: } 0.112\end{array}$ & $\begin{array}{c}\text { Error: 7.2e-4 } \\
\text { Shape: 1.088, Scale: } 1.60 \mathrm{e}-5 \\
\text { Location: } 1.39 \mathrm{e}-5\end{array}$ & $\begin{array}{l}\text { Error: } 9.7 \mathrm{e}-4 \\
\text { Scale: } 9.26 \mathrm{e}-5 \\
\text { Shape: } 0.32\end{array}$ & $\begin{array}{l}\text { Error: } 7.3 \mathrm{e}-4 \\
\text { Mean: }-10.473 \\
\text { SD: } 2.00\end{array}$ \\
\hline format convert & $\begin{array}{l}\text { Error: } 0.0696 \\
\text { Mean: } 0.015\end{array}$ & $\begin{array}{l}\text { Error: } 0.0073 \\
\text { Shape: } 0.123 \\
\text { Scale: } 0.121\end{array}$ & $\begin{array}{c}\text { Error: 6.5e-4 } \\
\text { Shape: 1.026, Scale: } 1.43 \mathrm{e}-5 \\
\text { Location: } 1.26 \mathrm{e}-5\end{array}$ & $\begin{array}{c}\text { Error: } 9.5 \mathrm{e}-4 \\
\text { Scale: } 7.77 \mathrm{e}-5 \\
\text { Shape: } 0.32\end{array}$ & $\begin{array}{l}\text { Error: } 6.8 \mathrm{e}-4 \\
\text { Mean: }-10.62 \\
\text { SD: } 1.94\end{array}$ \\
\hline CoMD & $\begin{array}{l}\text { Error: } 0.4073 \\
\text { Mean: } 0.00239\end{array}$ & $\begin{array}{l}\text { Error: } 0.2204 \\
\text { Shape: } 0.152 \\
\text { Scale: } 0.0157\end{array}$ & $\begin{array}{c}\text { Error: } 1.31 \mathrm{e}-2 \\
\text { Shape: } 0.7142, \text { Scale: } 1.0832 \mathrm{e}-\$ \\
\text { Location: } 1.079 \mathrm{e}-5\end{array}$ & $\begin{array}{l}\text { Error: } 1.57 \mathrm{e}-2 \\
\text { Scale: } 3.72 \mathrm{e}-5 \\
\text { Shape: } 0.3955\end{array}$ & $\begin{array}{l}\text { Error: 1.10e-2 } \\
\text { Mean: }-11.08 \\
\text { SD: } 1.67\end{array}$ \\
\hline MiniFE & $\begin{array}{c}\text { Error: } 0.5245 \\
\text { Mean: } 0.00389\end{array}$ & $\begin{array}{l}\text { Error: } 0.2084 \\
\text { Shape: } 0.140 \\
\text { Scale: } 0.0277\end{array}$ & $\begin{array}{c}\text { Error: } 6.5 \mathrm{e}-2 \\
\text { Shape: } 1.2634, \text { Scale: } 1.048 \mathrm{e}-5 \\
\text { Location: } 5.476 \mathrm{e}-6\end{array}$ & $\begin{array}{l}\text { Error: } 3.41 \mathrm{e}-2 \\
\text { Scale: } 7.04 \mathrm{e}-5 \\
\text { Shape: } 0.305\end{array}$ & $\begin{array}{l}\text { Error: } 3.37 \mathrm{e}-2 \\
\text { Mean: } \mathbf{- 1 1 . 0 7} \\
\text { SD: } 2.91\end{array}$ \\
\hline HPCCG & $\begin{array}{c}\text { Error: } 0.444 \\
\text { Mean: } 0.0029\end{array}$ & $\begin{array}{l}\text { Error: } 0.2100 \\
\text { Shape: } 0.154 \\
\text { Scale: } 0.0188\end{array}$ & $\begin{array}{c}\text { Error: } 4.4 \mathrm{e}-2 \\
\text { Shape: } 1.068, \text { Scale: } 1.4731 \mathrm{e}-5 \\
\text { Location: } 1.1441 \mathrm{e}-5\end{array}$ & $\begin{array}{l}\text { Error: } 3.58 \mathrm{e}-2 \\
\text { Scale: } 6.70 \mathrm{e}-5 \\
\text { Shape: } 0.345\end{array}$ & $\begin{array}{l}\text { Error: 3.91e-2 } \\
\text { Mean: } \mathbf{- 1 0 . 8 0} \\
\text { SD: } 2.17\end{array}$ \\
\hline
\end{tabular}

by the application to perform the required computation on $n$ nodes can be expressed as:

Total execution time $=S+P / n+O_{n}$,

where $S$ is the sequential part of the program, $P$ is the parallelable part, and $O_{n}$ is the communication overhead. There exists a trade-off between the degree of parallelism $(n)$ and the communication overhead $\left(O_{n}\right)$ incurred due to parallelism. As we increase $n$, the program runs more parallel but on the other hand we experience more communication overhead due to the required synchronization to merge the processed data. Figure 4 plots the execution time versus the number of nodes for the application under study (pagerank) under different dataset sizes. It can be seen that, in general, as we increase the size of the dataset, the performance keeps increasing for larger number of nodes. However, the overhead 


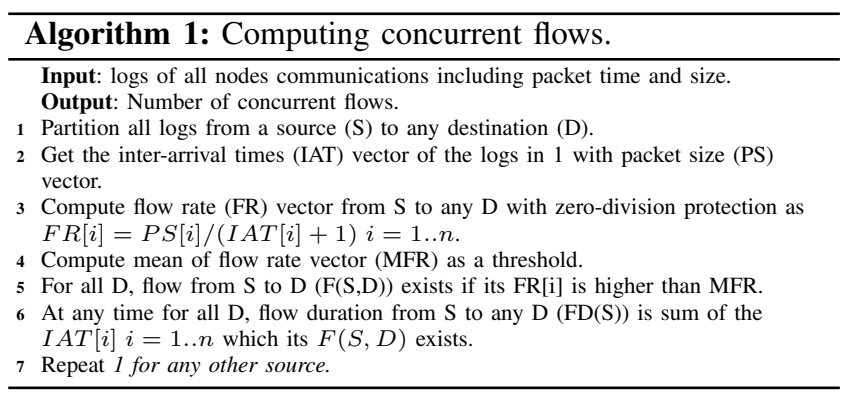

TABLE VII: The comparative time analysis of the maximum number of concurrent flows for studied workloads.

\begin{tabular}{|c|c|}
\hline Application & Concurrent Flows \\
\hline Data serving & 71 \\
\hline approximate diameter & 20 \\
\hline connected component & 42 \\
\hline directed triangle count & 54 \\
\hline format convert & 18 \\
\hline kcore & 75 \\
\hline pagerank & 28 \\
\hline undirected triangle count & 45 \\
\hline memcached & 1 \\
\hline CoMD & 40 \\
\hline MiniFE & 10 \\
\hline HPCCG & 5 \\
\hline
\end{tabular}

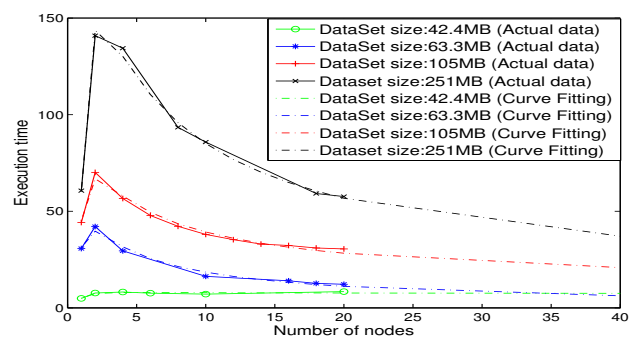

Fig. 4: Execution time versus the number of nodes for pagerank using default server architecture (architecture 1 in Table II).

of communication is too much for small dataset sizes. For dataset sizes less than $60 \mathrm{MB}$, as we increase the number of nodes, no performance improvement is achieved and there is no point in increasing the scale-out degree. Further, increasing the number of nodes (servers) from 1 to 2 degrades performance as the impact of the overhead is typically more pronounced with the small number of nodes. Using non-linear regression methods, the communication overhead in Equation 1 can be modeled as follows:

$$
O_{n}=\left(\alpha_{1}+\alpha_{2} / n\right) * \log (n) .
$$

where $\alpha_{1}$ represents the coefficient of the communication overhead of the sequential part (when one server forks the tasks for the other parallel servers), and $\alpha_{2}$ represents the coefficient of the communication overhead of the parallel part (when parallel servers want to join the processed data in one place). It is known that the implementation of the program has a logarithmic overhead [34]. Therefore, Equation 2 can accurately predict the execution time with respect to the datasize and the degree of parallelism. For small dataset sizes, the value of $\alpha_{1}$ is very close to zero and increases as we increase the dataset size. In addition, it is observed that, running the same dataset size

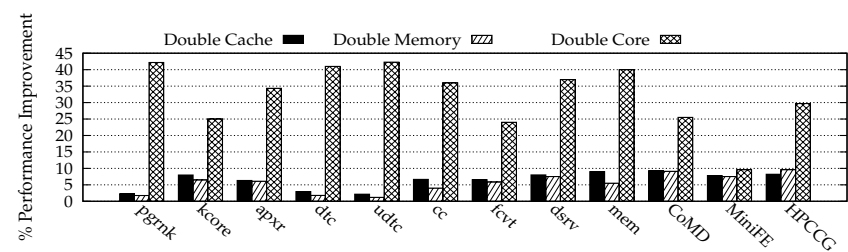

Fig. 5: Performance improvement of three different configurations with respect to the baseline.

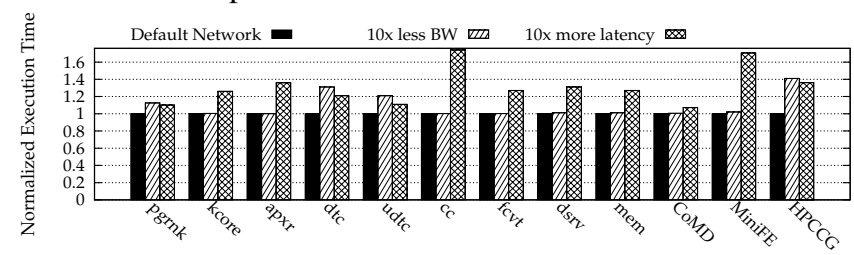

Fig. 6: Normalized execution time of three different network configurations with respect to the baseline.

on very large number of nodes increases the communication overhead such that beyond a certain point, parallelism level saturates and the communication overhead keeps increasing. Figure 4 plots the result of curve fitting and predicted tail (using dashed lines) using this model for pagerank and for different data sizes. The experiments in this figure show that, when doubling the dataset size, the overhead constant, $\alpha_{2}$, increases by $178 \%$; however, the parallel constant, $P$, in Equation 1, only increases by $50 \%$.

\section{B. Performance Comparison of Different Architectures}

To explore the impact of different node architectures, we evaluated the performance of different applications using the studied configurations, as was shown in Tables II and III, respectively. Figure 5 shows the performance improvement that each configuration gets with respect to the baseline system for different applications. The performance improvement is the average performance improvement for 10 different dataset sizes. It is observed that pagerank, approximate diameter, directed triangle count, undirected triangle count and connected components are applications which are less cache sensitive and get more performance improvement with more number of cores per server. On the other hand, kcore, CoMD, MiniFE and $H P C C G$ are more cache and memory sensitive and increasing the number of cores per server increases the contention for memory bandwidth. Therefore, these benchmarks show less performance improvement with more number of cores per server.

Further, based on the results of experiments with different network architectures, we can divide these applications into network bandwidth-intensive and network latency-sensitive categories. Figure 6 shows normalized execution time of the applications with different network configurations with respect to the baseline. It is observed that, MiniFE, HPCCG, approximate diameter and connected components are applications which are more sensitive to the network latency. On the other hand, pagerank, directed triangle count and undirected triangle count are bandwidth-intensive applications. Later, in section V-D, we show that the applications can have different performance/cost benefits in a scale-out or scale-up approach based on how network sensitive they are. 


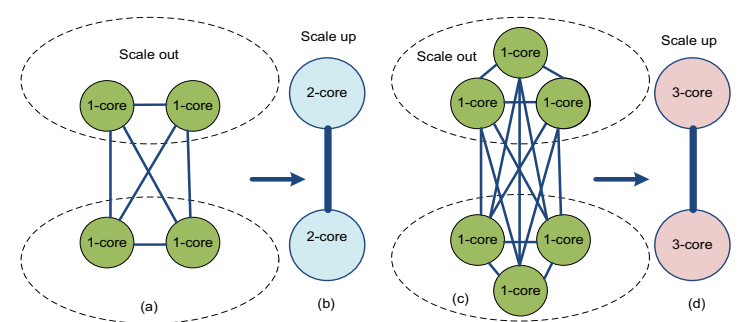

Fig. 7: Scale-up approach with two and three cores per node versus scale-out approach.

\section{Bandwidth Comparison between Scale-up and Scale-out}

In this part, we quantify the effect of different node architectures on the bandwidth requirement of our workloads. In particular, we target iso-core architectures, and compare the scale-up and scale-out approaches. Figures 7(a-b) and 7(cd) show 4 cores and 6 cores in the scale-out and scale-up configurations, respectively. Let us assume that a scale-up node is equal to packing $k$ cores in scale-out to construct a $k$-core scale-up machine as shown in Figure 7 . The virtual links in this figure show the communication demand between any two nodes in scale-out or scale-up configuration. In real configurations, nodes are not connected in a fully connected graphs, but the average bandwidth between two different nodes does not change. Let $S$ be the subset of links in the scaleout approach which connects the two parts of the graph, which are forming one vertical link in the scale-up approach. Investigating the different scale-out and scale-up approach with different number of nodes and different dataset sizes, we observed that if we aggregate $k$ nodes together the average communication bandwidth between any two k-core machines

is approximately:

In addition, the maximum bandwidth of each link in scale-up approach can be computed as follows:

$$
\max \left(B W_{\mathrm{k}-\text { core scale-up }}\right)=\max \left(\sum_{i=1, i \in S} B W_{\text {link \#i in scale-out }}\right)
$$

Figure 7 plots virtual links between any two communicating nodes in a scale-up approach which is $k^{2}$ times more than each link in the scale-out approach ${ }^{3}$. Using the proposed analytical approach, a designer can predict how much to increase the average bandwidth of network when scaling up server nodes. Since the maximum bandwidth in today's datacenter switches is $10 \mathrm{Gbps}$, scaling up the servers at some point may lead the network bandwidth be the bottleneck in the system.

Figure 8 shows the average bandwidth usage of different links in scale-out and scale-up approach for applications with east to west traffic patterns.

Our experiments also show that the web applications where

${ }^{3}$ The formula is derived using the fact that the average communication of each k-core machine should be approximately $k^{2}$ times more than the scale-out approach, however the total communication bandwidth is reduced by a factor of $\frac{k^{2}}{\left(\begin{array}{c}2 k \\ 2\end{array}\right)}$ which is the total number of outgoing links in scale-up approach over the total number of outgoing links in the scale-out approach. For example in a 2-core scale-up approach in Figure 3, every 4 link construct one link between two nodes in scale-up, and the total amount of off-chip communication is reduced by $4 / 6$ and in a 3 -core scale-up approach every 9 links in the scaleout construct one link in scale-up and the total off-chip communication is reduced by $9 / 15$.

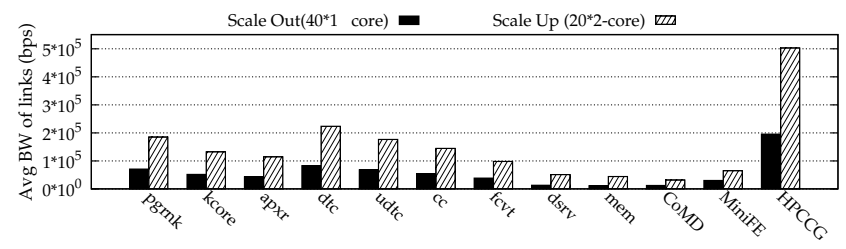

Fig. 8: Average bandwidth usage of different benchmarks for scale-up and scale-out approach.

a client sends request to servers, utilize the high level switches, regardless of mapping of cores to nodes, since there is very little communication among servers. However, in Map-reduce benchmarks, the traffic volume between different servers is very high; consequently, it would be better, if possible, to use the servers in the same rack to avoid traffic to be directed to high level switches.

\section{Performance Cost Analysis}

In this section, we evaluate performance/cost analysis of different applications in a scale-out and scale-up approach. To estimate the cost for datacenter in scale-out and scale-up approach, we assume a power usage effectiveness (PUE) of 1.7, and utility price of 0.07 dollar per kwh. As shown in Figure 5, most of cloud computing workloads are CPU and memory intensive. Thus, we assume CPU and memory price to be the major contributor of capital costs and power cost to be the major contributor of operating cost ${ }^{4}[13,35,36]$.

Figure 9 shows the total cost of ownership for different configurations with 192-cores in a scale-out or scale-up approach, using the offered cost of simulated AMD processors from [23]. For each configuration in Figure 9, represents the number of nodes, $B$ refers to the total number of cores inside a single node and $C$ is the number of sockets for each node. From total cost of ownership (TCO) perspective, it is observed that $16 \times 12$-core dual socket servers has the optimal TCO for the servers in a datacenter, but TCO does not consider workload's performance. Thus, we came up with a better metric to include TCO and performance together. Figure 10 shows speed up/cost with respect to one single core machine for different applications for scale-out and scaleup approaches. We assume iso-core, iso-cache capacity and iso-memory configurations. As it is seen in the figure, graph applications have little scalability and get better performance in scale-up approach. In fact, scale-up approach has better performance/cost for graph applications that needs to access random bits of data frequently. These applications are network bandwidth-intensive and the communication overhead of scaleout is abandons any hope to get the same performance as in the scale-up approach. But HPCCG as a bandwidth-intensive, latency-sensitive, and memory-intensive workload performs better in a scale-out approach. However, applications with high locality benefit from a scale-out approach. The total aggregated memory bandwidth of scale-out approach is higher than the scale-up, so memory intensive applications get more performance benefit from scale-out.

\footnotetext{
${ }^{4}$ For example, for a 96 dual-core scale-out approach, assuming Mean Time to Failure (MTTF) of three year (26280 hours), the total cost of ownership is $[95+26280 \times 0.07 \times 1.7 \times 65 / 1000] \times 96=\$ 28634$ where each dual core machine's CPU and memory cost is $\$ 95$ [23] and maximum power usage of each dual core machine is 65 watt [23]. The capital expense (CAPEX) of such configuration is $\$ 9120$ and the power cost $(\approx \mathrm{OPEX})$ is $\$ 19514$.
} 
TABLE VIII: Workload Classification.

\begin{tabular}{|l|l|l|l|l|l|l|l|l|l|l|l|l|}
\hline Characteristics & pgrnk & fcvt & udtc & dtc & kcore & cc & apxr & dsrv & mem & CoMD & MiniFE & HPCCG \\
\hline Network bandwidth-intensive & high & medium & high & high & medium & medium & medium & low & low & low & low & high \\
\hline Network latency-sensitive & low & medium & low & medium & medium & high & medium & medium & medium & low & high & high \\
\hline Memory-intensive & low & high & low & low & high & medium & medium & high & high & high & high & high \\
\hline $\begin{array}{l}\text { Required bisection bandwidth } \\
\text { (Concurrent flow) }\end{array}$ & medium & medium & high & high & high & high & medium & high & low & high & low & low \\
\hline Performance/TCO & $\begin{array}{c}\text { low } \\
\text { (scale-up) }\end{array}$ & $\begin{array}{c}\text { low } \\
\text { (scale-out) }\end{array}$ & $\begin{array}{c}\text { medium } \\
\text { (scale-up) }\end{array}$ & $\begin{array}{c}\text { low } \\
\text { (scale-up) }\end{array}$ & $\begin{array}{c}\text { low } \\
\text { (scale-out) }\end{array}$ & $\begin{array}{c}\text { low } \\
\text { (scale-up) }\end{array}$ & $\begin{array}{c}\text { medium } \\
\text { (scale-up) }\end{array}$ & $\begin{array}{c}\text { medium } \\
\text { (scale-out) }\end{array}$ & $\begin{array}{c}\text { medium } \\
\text { (scale-out) }\end{array}$ & $\begin{array}{c}\text { high } \\
\text { (scale-out) }\end{array}$ & $\begin{array}{c}\text { high } \\
\text { (scale-out) }\end{array}$ & $\begin{array}{c}\text { high } \\
\text { (scale-out) }\end{array}$ \\
\hline
\end{tabular}

TABLE IX: Implications of the metrics of interest.

\begin{tabular}{|l|l|l|l|l|}
\hline Metric & $\begin{array}{l}\text { graphlab (pagerank, directed triangle } \\
\text { count, undirected triangle count) }\end{array}$ & $\begin{array}{l}\text { graphlab (approximate diameter, } \\
\text { connected components) }\end{array}$ & $\begin{array}{l}\text { Mantevo (MiniFE, HPCCG, } \\
\text { CoMD) }\end{array}$ & $\begin{array}{l}\text { cloudsuite (memcached, data } \\
\text { serving) graphlab (kcore) }\end{array}$ \\
\hline Comm/Comp overhead & high & high & low \\
\hline Inter-arrival times & lognormal & gev & lognormal \\
\hline Packet Size Distribution & $\begin{array}{l}\text { Bimodal distribution with more large } \\
\text { messages }\end{array}$ & $\begin{array}{l}\text { Bimodal distribution with more } \\
\text { large messages }\end{array}$ & $\begin{array}{l}\text { Bimodal distribution with more } \\
\text { large messages }\end{array}$ & $\begin{array}{l}\text { multi modal distribution with } \\
\text { more smaller messages }\end{array}$ \\
\hline Bandwidth Requirement & large burst small duration flows & large burst small duration flows & large burst small duration flows & Small burst long duration flows \\
\hline Self Similarity & high high & hew \\
\hline Summary & $\begin{array}{l}\text { Network bandwidth-intensive with } \\
\text { better performance/cost for scale-up }\end{array}$ & $\begin{array}{l}\text { Network latency sensitive with bet- } \\
\text { ter performance/cost for scale-up }\end{array}$ & $\begin{array}{l}\text { Memory intensive with better per- } \\
\text { formance/cost for scale-out }\end{array}$ & $\begin{array}{l}\text { Stable flows, long duration } \\
\text { small packets, with better per- } \\
\text { formance/cost for scale-out }\end{array}$ \\
\hline
\end{tabular}

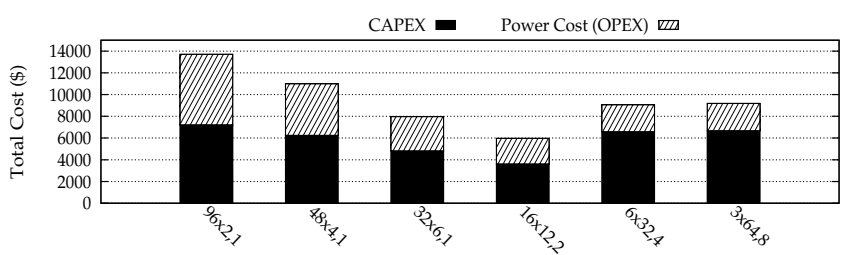

Fig. 9: Total cost of ownership for scale-out and scale-up approaches for various server types ("A $\times \mathrm{B}, \mathrm{C}$ " means A times B-core C-socket server).

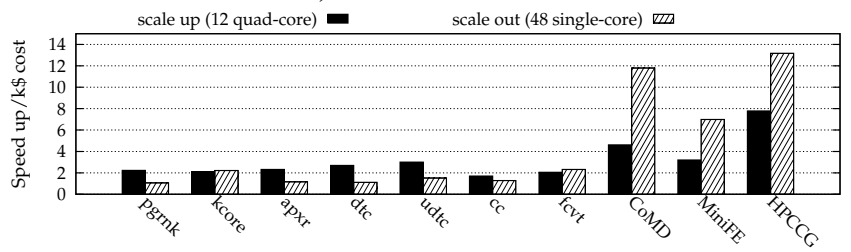

Fig. 10: Speed up per kilo dollar cost for scale-out (48 single core machines) and scale-up (12 quad-core machines).

\section{Potential IMPlications}

This paper studies the combined impact of node architecture and cloud workloads on network traffic. Based on our experimental results, one can reuse the results to analyze similar studied workloads. For example, inter-arrival times and packet size distribution can be used to regenerate the same traffic pattern. In addition, inter-arrival times imply a large amount of self-similarity. Based on our flow analysis, applications with high bandwidth demands and long-lived flows make the network buffer queues to grow faster until the packets are discarded. As a result, TCP congestion control is not fair for applications with low bandwidth demand and short-term flows. Our experimental results opens up the area of designing better congestion control mechanism and buffer managements for different classes of workloads.

Based on our observations, we classified the applications based on their bottleneck resources in Table VIII. Based on the results, applications are categorized into five different categories. It is observed that for the same number of cores, scale-out and scale-up approach can have completely different performance/cost metrics. The results can be used for system administrator to get use of heterogeneous designs for different applications. Table IX shows the implication of each metric of interest we discussed on each of the benchmarks we studied. The first class of benchmarks is network-bandwidth intensive benchmarks with better performance/cost for scale-up. The second class of benchmarks is more sensitive to the routing delay. The third class of benchmarks is memory-intensive applications, which get more memory resources in the scaleout approach. The last class of benchmarks have long duration flows with low burst, so these kind of traffics are more stable and are not sensitive to routing delay of high delay switches and can be routed through optical switches. In addition, we discussed how much bandwidth demand is increased when scaling up the server nodes and proposed an analytical model to predict network bandwidth demands of $k$-core servers. The results of this analysis can be used for network capacity planning. Knowing the characteristics of traffic behavior in advance, can enable one employ a hybrid network architecture, where long lived flows can be routed through optical switches and short duration flows use traditional electrical switches. Based on our experimental study, we can divide the benchmarks in our experimental suite into two groups of long-duration, lowburst flows and short-duration, high-burst traffics. Figure 11 plots two different benchmarks with different flow duration and bandwidth requirement. Figure 11a shows the bandwidth usage of two different server nodes, which shows a long short burst flow, that lives for the whole duration of running the benchmark. Figure 11b shows the aggregated bandwidth usage of all nodes during time, which shows short duration high burst flows. Classifying the network traffic into these groups can potentially help us in exploiting hybrid network architectures. For instance, we can take advantage of both optical switching and packet switching routers.

\section{RELATED WORK}

There are recent works aimed at improving the performance of datacenter networks to sustain huge amount of data communication between servers and with outside world. For 


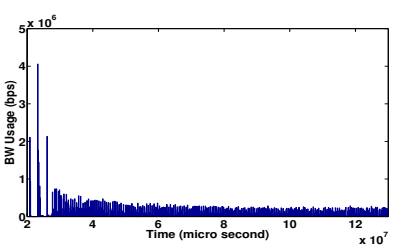

(a) kcore

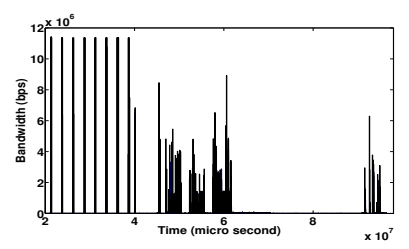

(b) directed triangle count
Fig. 11: Short burst long duration flow in kcore and high burst short duration flows in directed triangle count.

example $[10,11]$ suggest to use commodity switches in a fat tree topology for large-scale datacenters. However, managing routing algorithms to utilize the available bandwidth in such networks is very challenging and without a deep understanding of traffic and workload characteristic it is not possible to design a suitable routing algorithm for these kinds of networks.

While there are lots of works to improve the routing algorithms and network topology of datacenters, there is only few works to study the effect of node architecture on traffic pattern of datacenters. Benson et al in [9] have studied traffic behavior of 10 different datacenters using SNMP traces. The packet size distribution of different datacenters in this study follows a bimodal distribution, which is the same as what we found for most Map-Reduce applications. However using SNMP traces it is not possible to poll the switches very often so this study does not look into fine grain traffic behavior of different applications and does not study the effect node architecture on traffic behavior of datacenter networks. Ersoz et al [8] implemented a real 3-tier cluster-based datacenter, and characterize the network traffic behaviour of the nodes. They found that the distribution of inter-arrival times and message sizes of the incoming requests, conform lognormal distribution, and also Pareto distribution is probable for service times of the requests. However, they haven't studied inter-node communications among different servers. Chodnekar et al [37] characterize the network traffic behaviour of the interconnection network of a system when multiple parallel applications are running in the system. They investigate the distribution of message sizes and generation times as a common distribution using SPASM simulator with a dynamic and static strategies.

\section{CONCLUSION}

In this study, we conducted a workload characterization of wide range of modern cloud applications on a variety of node and network architectures. The results from this study can help us understand cost performance trade-offs in designing datacenters. One of the findings of our simulation-based study is that the inter-arrival times of packets follows a self-similar distribution and increasing the number of nodes tends to increase this self similarity. It is also shown that different benchmarks have different performance/cost for scale-out and scale-up approaches and changing hardware architecture, like CPU core architecture and memory hierarchy of a node can change the traffic pattern on the network and upgrading the servers without changing the network infrastructure, may lead the network to be the bottleneck in the system. We also observed that, some of the benchmarks send a high duration flow into the network, which makes them a suitable fit for hybrid datacenter networks where stable flows can pass through optical switches, whereas low-duration flows can pass through electrical switches.

\section{REFERENCES}

[1] J. Dean et al. Mapreduce: simplified data processing on large clusters. Communications of the ACM, 2008.

[2] K. H. Lee et al. Parallel data processing with mapreduce: a survey. ACM SIGMOD Record, 2012.

[3] S. M Rumble et al. It's time for low latency. In USENIX, HoOS, 2011.

[4] M. Alizadeh et al. Data center tcp. In SIGCOMM, 2011.

[5] D. G. Andersen et al. Fawn: A fast array of wimpy nodes. In ACM SIGOPS, 2009.

[6] V. J. Reddi et al. Web search using mobile cores: Quantifying and mitigating the price of efficiency. In ISCA, 2010.

[7] S. Kandula et al. The nature of data center traffic: Measurements \& analysis. In SIGCOMM, 2009.

[8] D. Ersoz et al. Characterizing network traffic in a cluster-based, multi-tier data center. In In ICDCS, 2007.

[9] T. Benson et al. Network traffic characteristics of data centers in the wild. In ACM SIGCOMM, 2010.

[10] M. Al-Fares et al. A scalable, commodity data center network architecture. In SIGCOMM, 2008.

[11] A. Vahdat et al. Scale-out networking in the data center. In Micro, 2010.

[12] C. Guo et al. Dcell: A scalable and fault-tolerant network structure for data centers. In ACM SIGCOMM, 2008.

[13] R. Appuswamy et al. Scale-up vs scale-out for hadoop: Time to rethink? In SoCC, 2013.

[14] http://parsa.epfl.ch/cloudsuite/.

[15] P. Lotfi-Kamran et al. Scale-out processors. In ISCA, 2012.

[16] M. Ferdman et al. Clearing the clouds: A study of emerging scale-out workloads on modern hardware. In SIGPLAN, 2012.

[17] http://mantevo.org/.

[18] http://graphlab.org/.

[19] J. Gonzalez et al. Y. Low. Graphlab: A new framework for parallel machine learning. In $C o R R, 2010$.

[20] L. Tang et al. The impact of memory subsystem resource sharing on datacenter applications. In ISCA, 2011.

[21] A. Khonsari et al. Mathematical analysis of buffer sizing for network-on-chips under multimedia traffic. In ICCD, 2008.

[22] http://www.amd.com/en-us/products.

[23] http://newegg.com/.

[24] http://cotson. sourceforge.net/.

[25] http://developer.amd.com/simnow-simulator/.

[26] E. Argollo et al. Cotson: Infrastructure for full system simulation. In ACM SIGOPS, 2009.

[27] http://snap.stanford.edu/data/.

[28] T. Schank. Algorithmic aspects of triangle-based network analysis. Phd thesis, University Karlsruhe, 2007.

[29] U. Kang et al. Hadi: Fast diameter estimation and mining in massive graphs with hadoop. Carnegie Mellon University, School of Computer Science, 2008.

[30] M. S. Taqqu et al. Estimators for long-range dependence: An empirical study. Fractals, 1995.

[31] W. E. Leland et al. On the self-similar nature of ethernet traffic. In IEEE/ACM Transactions on Networking, 1994.

[32] Z. Sahinoglu and S. Tekinay. On multimedia networks: selfsimilar traffic and network performance. In IEEE Communications Magazine, 1999.

[33] K. Park et al. Effect of traffic self-similarity on network performance. In IPCNS, 1997.

[34] J. Pješivac-Grbović et al. Performance analysis of mpi collective operations. Cluster Computing, 2007.

[35] D. Hardy et al. An analytical framework for estimating tco and exploring data center design space. ISPASS, 2013.

[36] C. Kozyrakis et al. Server engineering insights for large-scale online services. In MICRO, 2010.

[37] S. Chodnekar et al. Towards a communication characterization methodology for parallel applications. In HPCA, 1997. 